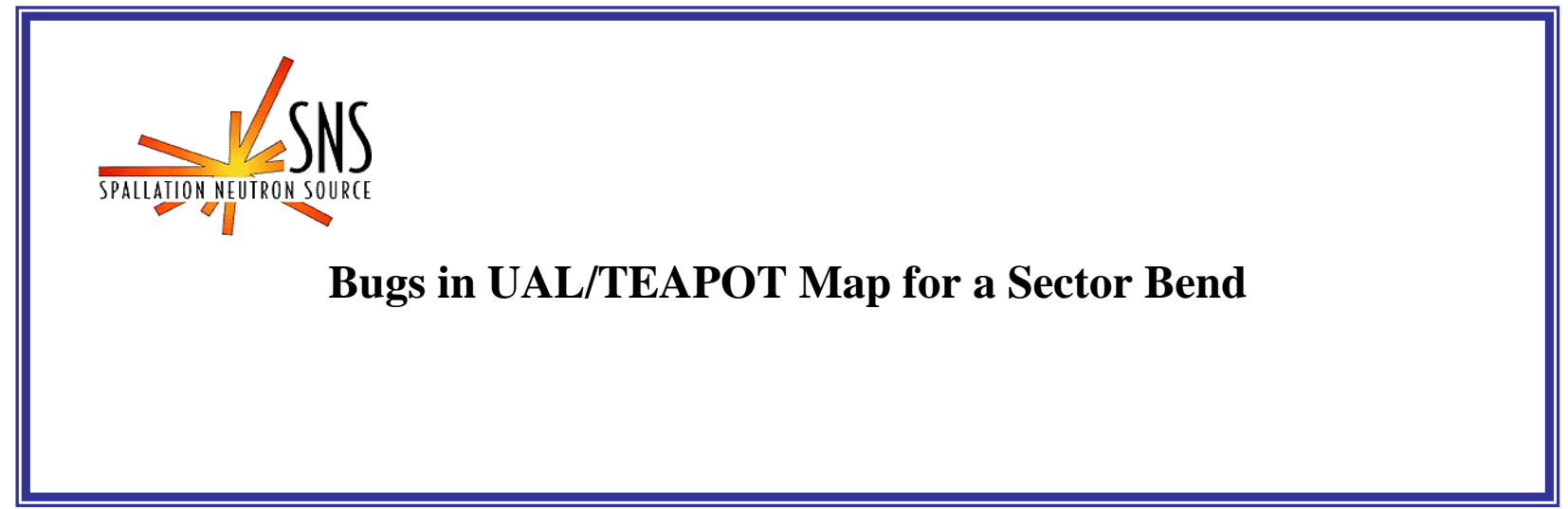

BNL/SNS TECHNICAL NOTE

NO. 073

Dan T. Abell

May 4, 2000

COLLIDER-ACCELERATOR DEPARTMENT

BROOKHAVEN NATIONAL LABORATORY

UPTON, NEW YORK 11973 


\title{
BUGS IN UAL/TEAPOT MAP FOR A SECTOR BEND
}

\author{
DAN T. ABELL
}

\begin{abstract}
Using simple geometric arguments, one can derive-and thereby check-some of the coefficients in the Taylor series map for a pure sector bend. This paper uses such arguments to show that the UAL/TEAPOT Taylor map for a sector bend contains terms not present in the actual solution.
\end{abstract}

\section{INTRODUCTION}

Tables 1 and 2 list the Taylor series coefficients for the transfer maps generated by respectively UAL/TEAPOT [2] and MARYLIE [1] for a pure sector bend. The maps agree to first order, but some of the second- and third-order coefficients differ quite dramatically. Using simple goemetric arguments, one can derive - and thereby check-some of those differing coefficients. This paper gives two such arguments that identify particular terms in the UAL/TEAPOT map as incorrect.

The codes UAL/TEAPOT and MARYLIE use the same set of canonical variables: $\left(x, p_{x}, y, p_{y}, \tau, p_{\tau}\right)$. Here $x$ and $y$ denote horizontal and vertical dispalcements from the design orbit, and $p_{x}$ and $p_{y}$ denote the corresponding conjugate momenta, scaled with respect to the design momentum, $p^{0}$. The variable $\tau=-c T$, where $c$ denotes the speed of light, and $T$ denotes the difference in flight times between the actual particle and one on the design orbit with the design momentum. And $p_{\tau}$ denotes the corresponding energy difference, scaled by $c p^{0}$. (In MaryLie the signs of $\tau$ and $p_{\tau}$ are actually the reverse of those used by UAL/TEAPOT, but this difference has no effect on the results described here.)

This paper looks at two aspects of the map for a pure sector bend: (i) the dependence of $\tau$ on $x$, and (ii) the dependence of $x$-final on $x$-initial. In both cases the map generated by UAL/TEAPOT contains terms not present in the actual solution.

\section{2. $\tau$ VERSUS $x$}

Consider a perfect sector bend with magnetic field $B$. It is designed to force a particle of charge $e$ and momentum $p$ to follow a circular path having a curvature radius $\rho=p / e B$. Such a magnet designed to bend particle trajectories by an angle $\theta$ has its entrance and exit faces inclined with respect to one another by exactly that angle $\theta$; then particles on the design orbit enter and exit this magnet normal to the end faces. Figure 1 illustrates this geometry and shows the design orbit in blue. Also shown, in red, is the trajectory of a particle whose initial conditions differ from the design orbit only by a horizontal displacement $x$ towards the outside of the magnet. This displaced particle trajectory has the same curvature as the design orbit, but its path length differs by the amount $\sigma$ indicated by the dotted line.

Date: 3 May 2000. 


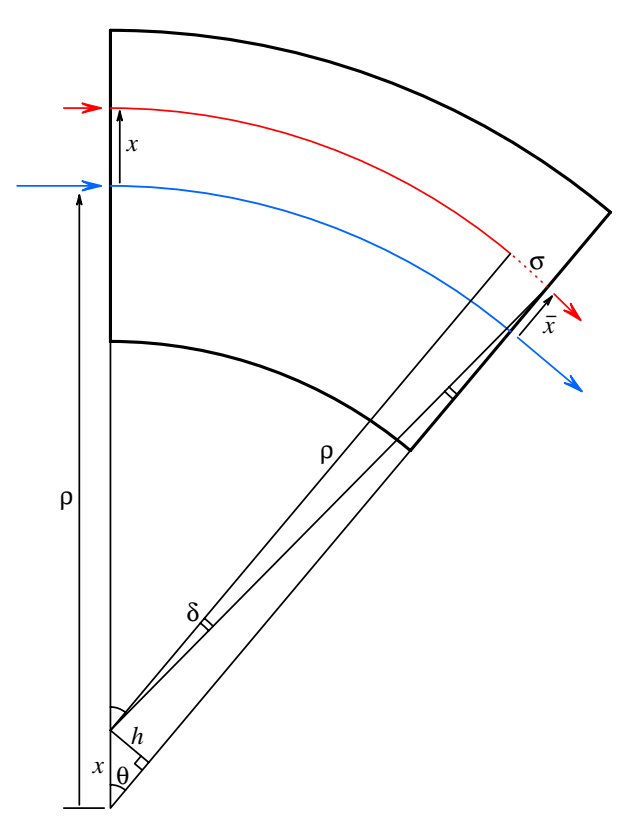

Figure 1. Geometry of pure sector bend.

The additional path length $\sigma$ subtends an angle $\delta=\sigma / \rho$ at its local center of curvature. We can relate $\delta$ to $x$ by noting that the distance $h$ in Figure 1 can be written in two different ways: $h=\rho \sin \delta$ and $h=x \sin \theta$. Hence

$$
\sin \delta=\frac{x}{\rho} \sin \theta
$$

It follows that $\tau$ is given by

$$
\tau=-c T=-c \frac{\sigma}{v}=-\frac{\sigma}{\beta}=-\frac{\rho \delta}{\beta}=-\frac{\rho}{\beta} \sin ^{-1}\left(\frac{x}{\rho} \sin \theta\right) .
$$

Expanding the arcsin in a power series yields the result

$$
\tau=-\frac{\sin \theta}{\beta} x-\frac{\sin ^{3} \theta}{6 \rho^{2} \beta} x^{3}-\cdots .
$$

Note in particular the absence of even powers of $x$, so that $\tau$ is odd in $x$.

The SNS dipoles have a length of $1.5 \mathrm{~m}$ and bend particles through $11.25^{\circ}$ along a curve of radius $7.64 \mathrm{~m}$. The relativistic ratio $\beta=0.875$ (at $1.0 \mathrm{GeV}$ ). Inserting these values into (3), we find

$$
\tau=-0.223 x-\left(2.42 \times 10^{-5}\right) x^{3}-\cdots .
$$

On the other hand, UAL/TEAPOT reports the $x$-dependence of $\tau$ as

$$
\tau=-0.223 x-6.10 x^{2}+0.798 x^{3}-\cdots .
$$

This expression not only has the wrong coefficient for the term cubic in $x$, but also includes a term quadratic in $x$. 


\section{3. $\bar{x}$ VERSUS $x$}

Now examine the relation between the horizontal deviations from the design orbit at the entrance and exit faces of our sector bend: respectively $x$ and $\bar{x}$. Referring again to Figure 1, we see that the distance from the magnet's center of curvature to the point at which our displaced orbit exits the magnet can be written in two different ways, which we equate:

$$
\rho+\bar{x}=x \cos \theta+\rho \cos \delta
$$

Writing $\cos \delta$ as $\sqrt{1-\sin ^{2} \delta}$, using (1), and expanding as a power series in $\mathrm{x}$, one finds

$$
\bar{x}=(\cos \theta) x-\frac{\sin ^{2} \theta}{2 \rho} x^{2}-\frac{\sin ^{4} \theta}{8 \rho^{3}} x^{4}-\cdots .
$$

Here notice that all terms other than the linear one are even in $x$. This result describes the well-known horizontal focusing caused by sector bends.

Inserting into (5) the values relevant for the SNS, we find

$$
\bar{x}=0.981 x-\left(2.49 \times 10^{-3}\right) x^{2}-\left(4.06 \times 10^{-7}\right) x^{4}-\cdots .
$$

On the other hand, UAL/TEAPOT reports the $x$-dependence of $\bar{x}$ as

$$
\bar{x}=0.981 x-\left(2.49 \times 10^{-3}\right) x^{2}-\left(1.46 \times 10^{-7}\right) x^{3}-\cdots,
$$

which includes a term cubic in $x$.

\section{Conclusions}

We have examined the Taylor map generated by UAL/TEAPOT for a pure sector bend and have identified particular terms that it contains which are not present in the actual solution, or which have incorrect coefficients. Since UAL generates maps using its tracking engine, this suggests that UAL's TEAPOT tracking engine does not track particles correctly through a sector bend.

The errors identified appear in both transverse and longitudinal motions. In the transverse case we have identified a third-order error. However, the maps shown in Tables 1 and 2 differ even at second order in the transverse variables, which suggests that UAL/TEAPOT's map for a sector bend is correct only to first order. On the other hand, since those errors appear to be small, they may not generate significant errors during the tracking of one thousand turns in the Spallation Neutron Source.

In the case of longitudinal (temporal) motion, we have identified a very large second-order error. The size of this error suggests that if time-dependent beamline elements (i.e., RF cavities) are turned on, then the corresponding UAL/TEAPOT tracking results cannot be trusted.

\section{ACKNOWLEDGEMENTS}

Nikolay Malitsky provided me with the UAL/TEAPOT Taylor map given in Table 1. And Frank Schmidt provided the initial insight that the Taylor series for $\tau$ should not contain a term proportional to $x^{2}$. 


\section{REFERENCES}

1. Alex J. Dragt, David R. Douglas, et al., MaryLie 3.0 User's Manual, University of Maryland, College Park, Physics Department Report, 1999.

2. Nikolay Malitsky and Richard Talman, Unified accelerator libraries, Computational Accelerator Physics, AIP Conference Proceedings No. 391 (Woodbury, NY) (Joseph J. Bisognano, , and Alfred A. Mondelli, eds.), AIP Press, 1997, pp. 337-342. 
Table 1: Taylor series coefficients in the map generated by $\mathrm{UAL} / \mathrm{TEAPOT}$ for a pure dipole sector magnet that bends $1 \mathrm{GeV}$ protons through $11.25^{\circ}$ along a $1.5 \mathrm{~m}$ path. The $j_{i}$ denote the exponents in the monomials $x^{j_{1}} p_{x}{ }^{j_{2}} y^{j_{3}} p_{y}{ }^{j_{4}} \tau^{j_{5}} p_{\tau}{ }^{j_{6}}$.

\begin{tabular}{|c|c|c|c|c|c|c|}
\hline$\overline{T_{j}}$ & $j_{1}$ & $j_{2}$ & $j_{3}$ & $j_{4}$ & $j_{5}$ & $j_{6}$ \\
\hline \multicolumn{7}{|l|}{\begin{tabular}{|l|}
$x$ \\
\end{tabular}} \\
\hline $9.80783 e-01$ & 1 & 0 & 0 & 0 & 0 & 0 \\
\hline $1.49030 \mathrm{e}+00$ & 0 & 1 & 0 & 0 & 0 & 0 \\
\hline $1.67771 \mathrm{e}-01$ & 0 & 0 & 0 & 0 & 0 & 1 \\
\hline$-2.49216 e-03$ & 2 & 0 & 0 & 0 & 0 & 0 \\
\hline $1.91370 e-01$ & 1 & 1 & 0 & 0 & 0 & 0 \\
\hline $4.35147 e-02$ & 1 & 0 & 0 & 0 & 0 & 1 \\
\hline $7.19915 e-02$ & 0 & 2 & 0 & 0 & 0 & 0 \\
\hline$-1.67067 e+00$ & 0 & 1 & 0 & 0 & 0 & 1 \\
\hline$-7.34022 e-02$ & 0 & 0 & 0 & 2 & 0 & 0 \\
\hline$-2.12413 e-01$ & 0 & 0 & 0 & 0 & 0 & 2 \\
\hline$-1.45685 e-07$ & 3 & 0 & 0 & 0 & 0 & 0 \\
\hline $7.28013 e-06$ & 2 & 1 & 0 & 0 & 0 & 0 \\
\hline $2.85192 e-03$ & 2 & 0 & 0 & 0 & 0 & 1 \\
\hline$-1.90290 e-02$ & 1 & 2 & 0 & 0 & 0 & 0 \\
\hline$-2.18829 e-01$ & 1 & 1 & 0 & 0 & 0 & 1 \\
\hline$-1.90382 e-02$ & 1 & 0 & 0 & 2 & 0 & 0 \\
\hline$-5.55895 e-02$ & 1 & 0 & 0 & 0 & 0 & 2 \\
\hline $7.30946 e-01$ & 0 & 3 & 0 & 0 & 0 & 0 \\
\hline $8.38635 e-02$ & 0 & 2 & 0 & 0 & 0 & 1 \\
\hline $7.30941 \mathrm{e}-01$ & 0 & 1 & 0 & 2 & 0 & 0 \\
\hline $2.13354 \mathrm{e}+00$ & 0 & 1 & 0 & 0 & 0 & 2 \\
\hline $2.50095 e-01$ & 0 & 0 & 0 & 2 & 0 & 1 \\
\hline $2.93715 e-01$ & 0 & 0 & 0 & 0 & 0 & 3 \\
\hline \multicolumn{7}{|l|}{$p_{x}$} \\
\hline$-2.55411 e-02$ & 1 & 0 & 0 & 0 & 0 & 0 \\
\hline $9.80783 e-01$ & 0 & 1 & 0 & 0 & 0 & 0 \\
\hline $2.22987 e-01$ & 0 & 0 & 0 & 0 & 0 & 1 \\
\hline$-4.97198 e-07$ & 2 & 0 & 0 & 0 & 0 & 0 \\
\hline$-7.48164 e-07$ & 1 & 1 & 0 & 0 & 0 & 0 \\
\hline $8.69089 e-06$ & 1 & 0 & 0 & 0 & 0 & 1 \\
\hline$-9.75602 e-02$ & 0 & 2 & 0 & 0 & 0 & 0 \\
\hline $5.43285 e-06$ & 0 & 1 & 0 & 0 & 0 & 1 \\
\hline$-9.75598 e-02$ & 0 & 0 & 0 & 2 & 0 & 0 \\
\hline$-2.98958 e-02$ & 0 & 0 & 0 & 0 & 0 & 2 \\
\hline$-4.28730 e-10$ & 3 & 0 & 0 & 0 & 0 & 0 \\
\hline $4.76874 e-08$ & 2 & 1 & 0 & 0 & 0 & 0 \\
\hline $5.79439 e-07$ & 2 & 0 & 0 & 0 & 0 & 1 \\
\hline$-7.53577 e-06$ & 1 & 2 & 0 & 0 & 0 & 0 \\
\hline $2.23056 e-08$ & 1 & 1 & 0 & 0 & 0 & 1 \\
\hline
\end{tabular}


Sector bend map coefficients (from UAL/TEAPOT)

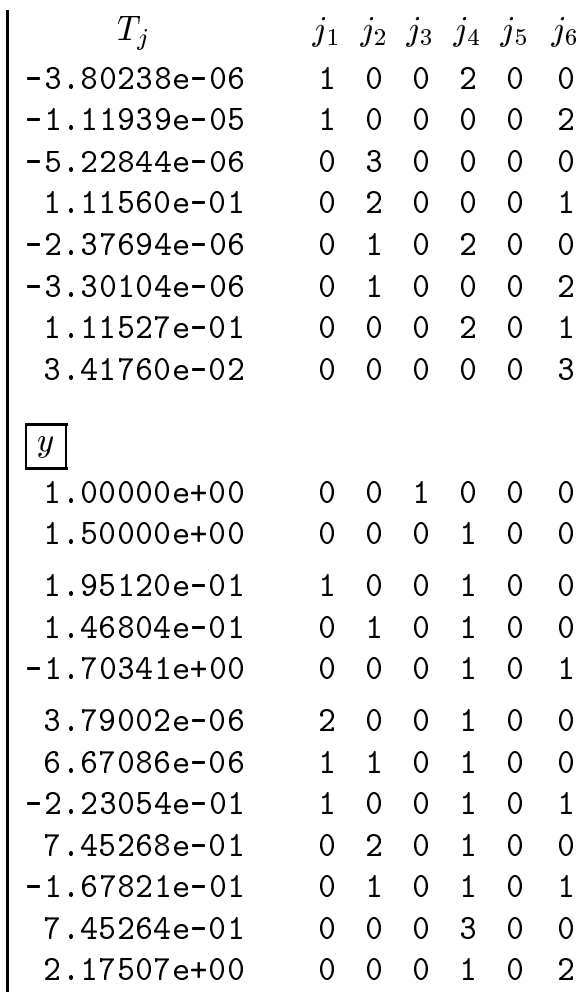

$$
\begin{aligned}
& p_{y} \\
& 1.00000 e+00 \quad 000001100 \\
& \tau \\
& -2.22987 e-01 \\
& -1.67771 \mathrm{e}-01 \\
& 1.00000 e+00 \\
& 4.46699 \mathrm{e}-01 \\
& -6.09506 e+00 \\
& -2.28566 e+00 \\
& 5.97913 e-02 \quad 1 \quad 0000001 \\
& -1.01242 e+00 \quad 0 \quad 2500000
\end{aligned}
$$

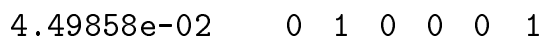

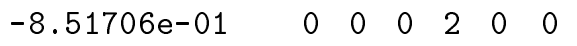

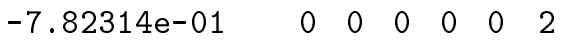

$$
\begin{aligned}
& 7.97857 \mathrm{e}-01 \quad 3 \quad 0 \quad 000000 \\
& \begin{array}{lllllll}
7.75943 e-02 & 2 & 1 & 0 & 0 & 0 & 0
\end{array} \\
& 1.63289 \mathrm{e}+00 \quad 2 \quad 0 \quad 0000001 \\
& -1.28354 e-01 \quad 1 \quad 2 \quad 0 \quad 0000
\end{aligned}
$$

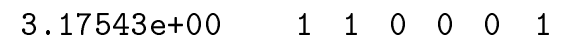

$$
\begin{aligned}
& \begin{array}{lllllll}
-1.11527 e-01 & 1 & 0 & 0 & 2 & 0 & 0 \\
-1.08071 \mathrm{e}-01 & 1 & 0 & 0 & 0 & 0 & 2
\end{array}
\end{aligned}
$$


Sector bend map coefficients (from UAL/TEAPOT)

\begin{tabular}{|ccccccc|}
$T_{j}$ & $j_{1}$ & $j_{2}$ & $j_{3}$ & $j_{4}$ & $j_{5}$ & $j_{6}$ \\
$-8.43063 \mathrm{e}-02$ & 0 & 3 & 0 & 0 & 0 & 0 \\
$2.54868 \mathrm{e}+00$ & 0 & 2 & 0 & 0 & 0 & 1 \\
$-8.39107 \mathrm{e}-02$ & 0 & 1 & 0 & 2 & 0 & 0 \\
$1.35766 \mathrm{e}-01$ & 0 & 1 & 0 & 0 & 0 & 2 \\
$2.17507 \mathrm{e}+00$ & 0 & 0 & 0 & 2 & 0 & 1 \\
$1.27818 \mathrm{e}+00$ & 0 & 0 & 0 & 0 & 0 & 3 \\
$p_{\tau}$ & & & & & & \\
$1.00000 \mathrm{e}+00$ & 0 & 0 & 0 & 0 & 0 & 1 \\
\hline
\end{tabular}


Table 2: Taylor series coefficients in the map generated by MARYLIE for a pure dipole sector magnet that bends $1 \mathrm{GeV}$ protons through $11.25^{\circ}$ along a $1.5 \mathrm{~m}$ path. The $j_{i}$ denote the exponents in the monomials $x^{j_{1}} p_{x}^{j_{2}} y^{j_{3}} p_{y}{ }^{j_{4}} \tau^{j_{5}} p_{\tau}{ }^{j_{6}}$. (This map has been adjusted to conform to the sign conventions used by UAL/TEAPOT for the variables $\tau$ and $p_{\tau}$.)

\begin{tabular}{|c|c|c|c|c|c|c|}
\hline$T_{j}$ & $j_{1}$ & $j_{2}$ & $j_{3}$ & $j_{4}$ & $j_{5}$ & $j_{6}$ \\
\hline$x$ & & & & & & \\
\hline $9.80785 e-01$ & 1 & 0 & 0 & 0 & 0 & 0 \\
\hline $1.49038 e+00$ & 0 & 1 & 0 & 0 & 0 & 0 \\
\hline $1.67755 e-01$ & 0 & 0 & 0 & 0 & 0 & 1 \\
\hline$-2.49104 e-03$ & 2 & 0 & 0 & 0 & 0 & 0 \\
\hline $1.91342 e^{-01}$ & 1 & 1 & 0 & 0 & 0 & 0 \\
\hline $4.34961 e-02$ & 1 & 0 & 0 & 0 & 0 & 1 \\
\hline $7.19846 e-02$ & 0 & 2 & 0 & 0 & 0 & 0 \\
\hline$-1.67051 e+00$ & 0 & 1 & 0 & 0 & 0 & 1 \\
\hline$-7.33948 e-02$ & 0 & 0 & 0 & 2 & 0 & 0 \\
\hline$-2.12334 e-01$ & 0 & 0 & 0 & 0 & 0 & 2 \\
\hline $2.84682 e-03$ & 2 & 0 & 0 & 0 & 0 & 1 \\
\hline$-1.90301 e-02$ & 1 & 2 & 0 & 0 & 0 & 0 \\
\hline$-2.18670 e-01$ & 1 & 1 & 0 & 0 & 0 & 1 \\
\hline$-1.90301 e-02$ & 1 & 0 & 0 & 2 & 0 & 0 \\
\hline$-5.55325 e-02$ & 1 & 0 & 0 & 0 & 0 & 2 \\
\hline $7.30872 e-01$ & 0 & 3 & 0 & 0 & 0 & 0 \\
\hline $8.38773 e-02$ & 0 & 2 & 0 & 0 & 0 & 1 \\
\hline $7.30872 e-01$ & 0 & 1 & 0 & 2 & 0 & 0 \\
\hline $2.13278 e+00$ & 0 & 1 & 0 & 0 & 0 & 2 \\
\hline $2.50020 e-01$ & 0 & 0 & 0 & 2 & 0 & 1 \\
\hline $2.93508 e-01$ & 0 & 0 & 0 & 0 & 0 & 3 \\
\hline \multicolumn{7}{|l|}{$p_{x}$} \\
\hline$-2.55373 e-02$ & 1 & 0 & 0 & 0 & 0 & 0 \\
\hline $9.80785 e-01$ & 0 & 1 & 0 & 0 & 0 & 0 \\
\hline $2.22954 e-01$ & 0 & 0 & 0 & 0 & 0 & 1 \\
\hline$-9.75452 e-02$ & 0 & 2 & 0 & 0 & 0 & 0 \\
\hline$-9.75452 e-02$ & 0 & 0 & 0 & 2 & 0 & 0 \\
\hline$-2.98533 e-02$ & 0 & 0 & 0 & 0 & 0 & 2 \\
\hline $1.11477 e-01$ & 0 & 2 & 0 & 0 & 0 & 1 \\
\hline $1.11477 e-01$ & 0 & 0 & 0 & 2 & 0 & 1 \\
\hline $3.41171 \mathrm{e}-02$ & 0 & 0 & 0 & 0 & 0 & 3 \\
\hline \multicolumn{7}{|l|}{$y$} \\
\hline $1.00000 e+00$ & 0 & 0 & 1 & 0 & 0 & 0 \\
\hline $1.50000 \mathrm{e}+00$ & 0 & 0 & 0 & 1 & 0 & 0 \\
\hline $1.95090 \mathrm{e}-01$ & 1 & 0 & 0 & 1 & 0 & 0 \\
\hline $1.46790 \mathrm{e}-01$ & 0 & 1 & 0 & 1 & 0 & 0 \\
\hline
\end{tabular}


BUGS IN UAL/TEAPOT MAP FOR A SECTOR BEND

Sector bend map coefficients (from MaryLie)

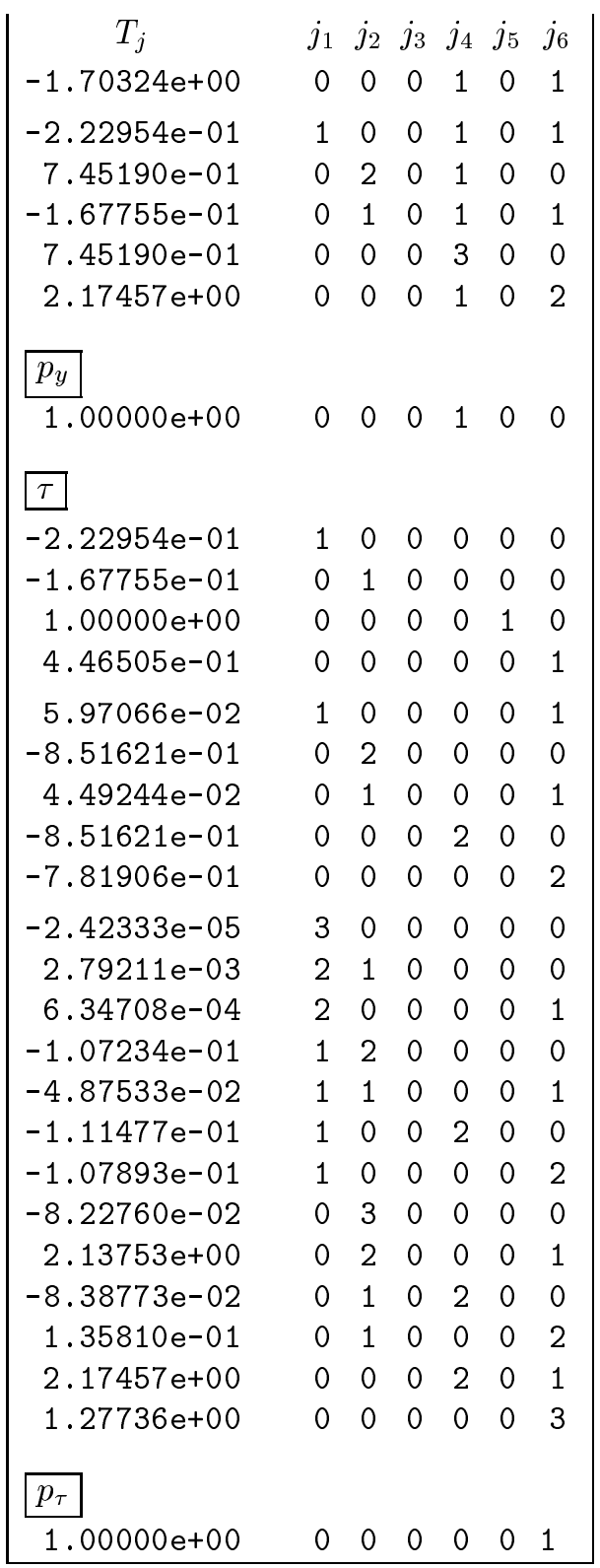

Collider-Accelerator Department, Brookhaven National Laboratory, Upton Ny $11973-5000$

E-mail address: dabell@bnl.gov 